\title{
MÉTODO DE TRABALHO DE ENFERMAGEM VOLTADO A PREVENÇÃo E TRATAMENTO DE ERROS ASSISTENCIAIS
}

\author{
Giselly Alves Lopes ${ }^{1}$ \\ Andreia Bilaque $^{2}$ \\ Mariana Morales Oliveira ${ }^{3}$ \\ Marcio Antônio de Assis ${ }^{4}$ \\ Maria Aparecida Xavier Moreira da Silva ${ }^{5}$
}

Resumo: Uma assistência efetiva necessita que os profissionais presem a segurança do paciente por isso é essencial o conhecimento da prevenção e notificação do evento diminuindo assim os riscos assistenciais. Tratou-se de uma pesquisa de campo do tipo descritiva realizada em uma instituição hospitalar em Guararema, São Paulo. Em visita a instituição de saúde foi constatado os riscos, sendo eles: broncoaspiração, erro de medicação, flebite, queda e ulcera por pressão, eles foram descrito em um mapeamento de riscos, juntamente com o protocolo de prevenção dos erros assistenciais e a elaboração de um instrumento de notificação associado ao fluxo que o mesmo apresentava na instituição. Esse estudo descreveu um método de trabalho de enfermagem voltado à prevenção e tratamento de erros assistenciais junto ao paciente e mostrou a necessidade de um bom preparo da equipe para que a qualidade da assistência prestada ao indivíduo seja com foco na prevenção de erros.

Palavras-chave: Avaliação em enfermagem; Planejamento de assistência ao paciente; Segurança do paciente.

\footnotetext{
${ }^{1}$ Enfermagem/Universidade de Mogi das Cruzes, Brasil. E-mail: gi_alvees@hotmail.com.

2 Enfermagem/Universidade de Mogi das Cruzes, Brasil. E-mail: andreia_bilac@hotmail.com.

3 Enfermagem/Universidade de Mogi das Cruzes, Brasil. E-mail: mari_morales_oliveira@hotmail.com.

${ }^{4}$ Enfermagem/Universidade de Mogi das Cruzes, Brasil. E-mail: marcioassis80@gmail.com.

${ }^{5}$ Enfermagem/Universidade de Mogi das Cruzes, Brasil. E-mail: maria.silva@umc.br.
} 